\title{
Specialist follow up of patients before end stage renal failure and its relationship to survival on dialysis
}

\author{
J Stoves, C N Bartlett, C G Newstead
}

\begin{abstract}
The high mortality rate of patients with end stage renal failure (ESRF) treated by dialysis is determined principally by irreversible factors such as age and comorbidity. In this single centre retrospective study of all 1260 ESRF patients who started dialysis between 1980 and 1999 it has been demonstrated that a short duration of specialist predialysis follow up is associated with a worse long term outcome on dialysis.
\end{abstract}

Kaplan-Meier survival curves were plotted according to duration of predialysis follow up (group $A, \leqslant 90$ days; group B $>90$ days), censoring for first transplant, and compared using a log rank test. Differences between groups were examined using an unpaired $t$ test. Cox regression analysis was performed to examine the influence of selected variables on survival.

Group A had the worst mortality (survival proportions of $87 \%, 74 \%$, and $31 \%$ in $A$ and $94 \%, 87 \%$, and $55 \%$ in $B$ at four months, one year, and five years respectively, $p<0.001)$. The increased risk of death was seen principally during the first few months of dialysis. ESRF associated with systemic disease was more prevalent in A. There were small but significant differences in predialysis clinical data, including age and serum albumin $(p<0.001)$. Fewer patients in $A$ were suitable for transplant listing $(\mathbf{p}<0.01)$. In the regression analysis, age, diabetes, predialysis serum albumin, suitability for transplant work-up and listing ("transplantability"), and the interval between referral and dialysis were significant predictors of survival.

In summary, this study strengthens the previously reported association between late referral of ESRF patients and subsequent poor survival on dialysis. This important message is relevant to all potential referring physicians.

(Postgrad Med f 2001;77:586-588)

Keywords: dialysis; survival; delayed referral; end stage renal failure

Dialysis treatment can offer good quality rehabilitation to patients with end stage renal failure (ESRF). Overall, however, there is premature mortality. The average remaining lifespan of patients starting dialysis between the ages of 39 and 44 years in the United States is estimated to be only 10 years. ${ }^{1}$ A recent longitudinal study from the Renal Data System Coordinating Centre in the United States examined the risk of mortality in over 200000 patients with ESRF. The annual death rate for all patients on dialysis was 16.1 per 100 patient years, although a lower rate of 6.3 per 100 patient years was seen in those listed for transplantation. ${ }^{2}$ For a 1997 ESRF cohort in the UK (representing a different case mix in terms of ethnicity and comorbidity), the unadjusted one year death rate for patients remaining on dialysis beyond the first 90 days was 19.3 per 100 patient years. $^{3}$

Age and non-renal comorbidity are the main determinants of survival in ESRF, but male gender, white race, malnutrition, and suboptimal dialysis dose are also reported to independently predict mortality. ${ }^{4-7}$ Of the modifiable factors that may influence outcome, the quality of predialysis medical care has received attention, as part of the debate about when renal support should be initiated. ${ }^{8-10}$ Patients who are referred with advanced chronic renal failure have lower estimates of lean body mass and more pronounced uraemia, anaemia, acidosis, anaemia, and hypoalbuminaemia at the time dialysis is commenced. ${ }^{11-13}$ The impact of late referral on mortality has been explored in only a few studies, involving relatively small numbers of patients. In the study reported here, the association between length of predialysis follow up and subsequent survival on dialysis is further explored in a large group of patients with ESRF.

\section{Patients and methods}

The Department of Renal Medicine at St James's University Teaching Hospital is a regional centre that currently provides dialysis facilities for more than 300 patients. The unit's electronic database was used to review the records of 1260 new patients with ESRF who had received dialysis treatment between 1980 and the date of census (July 1999). No patients were lost to follow up. The following data were recorded for each case: date of referral to the unit, date of first dialysis, and age when treatment was started. Additional data concerning primary renal disease, laboratory blood values just before the initiation of dialysis (haemoglobin, calculated creatinine clearance, albumin, bicarbonate, phosphate), blood pressure, length of survival, and cause of death were recorded when available. Kaplan-Meier survival curves were produced for patients grouped by the duration of predialysis follow up (group A, 0-90 days, $\mathrm{n}=467$; group $\mathrm{B}$, 


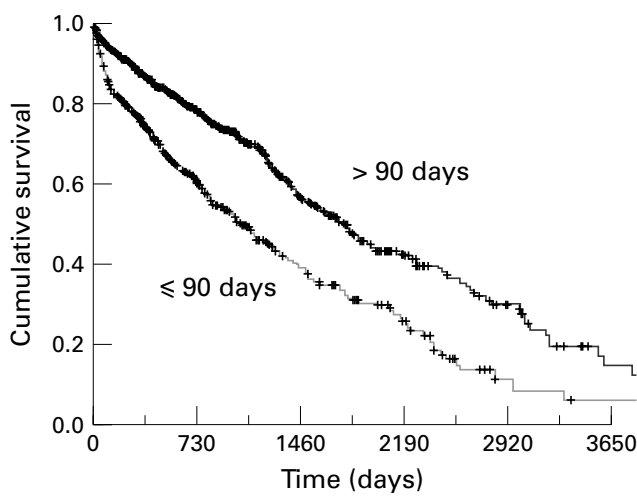

Figure 1 Survival curves for the two groups of patients (crosses mark points of censor).

longer than 90 days, $\mathrm{n}=793)$, censoring for first transplant $(n=496)$, and transfer away from the unit $(n=43)$. Differences between groups were examined using an unpaired $t$ test. Survival curves were compared using a log rank test. A Cox regression survival analysis was performed to examine the influence of selected variables, including "transplantability" (SPSS statistical software, version 9.0, Chicago, USA). Transplantability refers to a subjective assessment of a patient's suitability for transplant work-up and listing, based on a review of

Table 1 Cause of ESRF in the two patient groups; results are number (\%)

\begin{tabular}{lcc}
\hline & $\begin{array}{c}0-90 \text { Days follow up } \\
(n=467)\end{array}$ & $\begin{array}{l}>90 \text { Days follow up } \\
(n=793)\end{array}$ \\
\hline Primary glomerulonephritis & $37(8)$ & $141(18)$ \\
Interstitial nephropathy/pyelonephritis & $52(11)$ & $115(15)$ \\
Congenital/hereditary renal disease & $18(4)$ & $133(17)$ \\
Diabetic renal disease & $42(9)$ & $67(8)$ \\
Other secondary glomerulopathies/ & & $163(21)$ \\
$\quad$ vasculopathies/systemic diseases & $139(30)$ & $18(2)$ \\
Miscellaneous & $20(4)$ & $135(17)$ \\
Uncertain & $134(29)$ & $21(3)$ \\
Unrecorded & $25(5)$ & \\
\hline
\end{tabular}

Table 2 Recorded cause of death in the two patient groups; results are number (\%)

\begin{tabular}{lcc}
\hline & $\begin{array}{l}0-90 \text { Days follow up } \\
(n=254)\end{array}$ & $\begin{array}{l}>90 \text { Days follow up } \\
(n=333)\end{array}$ \\
\hline Cardiac & $77(30)$ & $129(39)$ \\
Vascular & $32(13)$ & $35(10)$ \\
Infection & $54(21)$ & $69(21)$ \\
Liver disease & 0 & $2(1)$ \\
Gastrointestinal & $5(2)$ & $9(3)$ \\
Discontinuation of therapy & $25(10)$ & $24(7)$ \\
Miscellaneous & $53(24)$ & $64(18)$ \\
Accident & 0 & $1(1)$ \\
\hline
\end{tabular}

Table 3 Patient data at the start of dialysis

\begin{tabular}{|c|c|c|c|c|c|}
\hline & $\begin{array}{l}\text { 0-90 Days } \\
\text { follow up }\end{array}$ & No & $\begin{array}{l}>90 \text { Days } \\
\text { follow up }\end{array}$ & No & $p$ Value \\
\hline Age & $55.2(18.1)$ & 467 & $51.1(16.7)$ & 793 & $\mathrm{p}<0.001^{\star}$ \\
\hline Gender (M:F ratio) & $1.58: 1$ & 467 & $1.52: 1$ & 793 & NS† \\
\hline \multicolumn{6}{|l|}{ Start of dialysis (No (\%)) } \\
\hline $1980-85$ & $50(11)$ & & $102(13)$ & & \\
\hline $1985-90$ & $103(22)$ & & $188(24)$ & & NSt \\
\hline $1990-95$ & $177(38)$ & & $262(33)$ & & \\
\hline 1995-census & $137(29)$ & & $241(30)$ & & \\
\hline Mode of dialysis (HD:PD ratio) & $5.7: 1$ & & $5.3: 1$ & & NSt \\
\hline Haemoglobin $(\mathrm{g} / \mathrm{l})$ & $83(17)$ & 325 & $84(18)$ & 704 & $\mathrm{NS}^{\star}$ \\
\hline Serum albumin $(g / 1)$ & $34.2(5.7)$ & 309 & $37.9(6.0)$ & 679 & $\mathrm{p}<0.001^{\star}$ \\
\hline Serum bicarbonate $(\mathrm{mmol} / \mathrm{l})$ & $20.5(4.9)$ & 284 & $19.6(4.8)$ & 627 & $\mathrm{p}<0.05^{\star}$ \\
\hline Serum phosphate $(\mathrm{mmol} / \mathrm{l})$ & $2.17(0.8)$ & 290 & $2.30(0.74)$ & 636 & $\mathrm{p}<0.05^{\star}$ \\
\hline Systolic blood pressure (mm Hg) & $147(26)$ & 178 & $153(24)$ & 489 & $\mathrm{p}<0.01^{\star}$ \\
\hline Diastolic blood pressure ( $\mathrm{mm} \mathrm{Hg}$ ) & $79(14)$ & 178 & $83(14)$ & 489 & $\mathrm{p}<0.01^{\star}$ \\
\hline Transplantability (\%) & 45.0 & 404 & 61.4 & 729 & $\mathrm{p}<0.001 \dagger$ \\
\hline
\end{tabular}

*Unpaired two tailed $t$ test.

$+\chi^{2}$ test. HD:PD haemodialysis:peritoneal dialysis. comorbid factors, that is made by senior clinicians in our unit during the first six months of dialysis treatment.

\section{Results}

The interval between first contact with the renal unit and start of dialysis was 90 days or less in approximately one third of patients (group A). Figure 1 shows that patients in this group had a significantly poorer survival compared with patients with longer follow up (group B, p<0.001). The cumulative survival at three months, one year and five years after initiation of dialysis was $87 \%, 74 \%$, and $31 \%$ in group A and $94 \%, 87 \%$, and $55 \%$ in group B. It is notable that most of the survival disadvantage occurred in the first four months after starting dialysis treatment.

Tables 1 and 2 list recorded primary renal disease and cause of death for the groups $\mathrm{A}$ and B, coded according to the European Dialysis and Transplant Association convention. There were more patients with primary glomerulonephritis and adult polycystic kidney disease $(31 \% v 11 \%, \mathrm{p}<0.001)$ in group $\mathrm{B}$, but the prevalence of diabetes mellitus in A and B was similar ( $9 \%$ v 10\%, NS).

Table 3 summarises laboratory data and blood pressure that were recorded before initiation of dialysis treatment for the two patient groups. There were small but significant differences in predialysis clinical data, including age (mean (SD) 55.2 (18.1) years in $\mathrm{A} v 51.1$ (16.7) years in $\mathrm{B}, \mathrm{p}<0.001)$ and serum albumin (34 (6) in A $v 38$ (6) in $\mathrm{B}, \mathrm{p}<0.001$ ). Fewer patients in A were suitable for transplant listing $(45.0 \% v 61.4 \%, \mathrm{p}<0.01)$. It is relevant to note that the proportion of patients in different age strata who were judged to be suitable for transplant listing remained stable during the 20 year period that was studied, suggesting that there was a consistency of approach to the assessment.

In the multifactorial regression analysis, age (hazard ratio (HR) 1.03 (0.005) per additional year, $\mathrm{p}<0.001$ ), diabetes (HR 1.56 (0.175), $\mathrm{p}<0.02$ ), predialysis serum albumin (HR 1.05 (0.009) per $1 \mathrm{~g} / 1$ decrement, $\mathrm{p}<0.001)$, suitability for transplant work-up and listing ("transplantability", HR 2.08 (0.143), $\mathrm{p}<0.001$ ), and the interval between referral and dialysis (HR 1.43 (0.115) for group A compared with group $\mathrm{B}, \mathrm{p}<0.001)$ were identified as significant predictors of survival.

\section{Discussion}

This review of our own experience over two decades further strengthens the association between late referral and increased mortality on dialysis, most noticeably during the first months of treatment. This is partly attributable to a higher level of comorbidity in patients who are referred late, as suggested by the lower proportion of patients who were judged to be suitable for transplantation and the higher prevalence of renal failure associated with systemic diseases in our late referral group. However, the Cox regression model suggests that a relatively short period of specialist predialysis follow up 
is an additional independent factor contributing to increased mortality.

The relevance of duration of follow up to outcome has been explored in a few studies involving relatively small numbers of patients. In a prospective study by Sesso and Belasco of 184 patients with ESRF, survival beyond the first six months of dialysis for patients referred less than a month before treatment was $69 \%$, compared with $87 \%$ for patients whose predialysis follow up period exceeded three months. ${ }^{14}$ In a British study of 44 patients with ESRF who died during the first year of dialysis, the average duration of predialysis follow up was much shorter than for matched controls who survived beyond the first year ( 36 days $v$ 30 months)..$^{15}$

Although any definition of late referral is arbitrary, there is considerable evidence from the literature that many patients start renal replacement therapy having had little prior contact with renal services. In a series that is fairly typical of referral patterns in well developed health care systems, $22 \%$ of patients starting dialysis for ESRF in the United States had received less than four months of specialist follow up. ${ }^{4}$ A similarly high rate of late referral has been reported in other American studies. ${ }^{16}$ In one study from New York, $57 \%$ of patients had not received predialysis care from a nephrologist. ${ }^{17}$ In a Brazilian study of 252 patients with ESRF, $42 \%$ had been diagnosed within a month of starting renal replacement therapy. ${ }^{14}$ A French study found that only 191 out of 256 patients $(75 \%)$ had been seen for more than six months before dialysis. ${ }^{11}$

The data from the large series we report are consistent with those seen in other well developed health care systems. The proportion of patients referred late was similar throughout the 20 year period covered by the study. The implication may be that the importance of specialist follow up of patients before ESRF has not been given sufficient emphasis in communications with primary care physicians and hospital colleagues from other departments. It is perhaps surprising that the majority of published reports concerning delayed referral have appeared in specialist journals.

It is received wisdom that the preparation of patients for renal replacement therapy should include the education of the patient and immediate family, establishment of appropriate dialysis access, and treatment of anaemia, hyperparathyroidism, hypertension, and malnutrition. These goals may not be achieved if referral is delayed. ${ }^{8}$ Many would regard the arbitrary 90 day interval that we have selected as being a minimum for adequate assessment and planning of treatment.

In summary, the evidence from this large retrospective study and other reports suggests that the association between prior specialist assessment and long term outcome is not attributable simply to differences in casemix and comorbidity, factors that are only marginally influenced by medical care. ${ }^{15}$ Delays in the

\section{Key points}

- The high mortality rate of patients with ESRF treated by dialysis is determined principally by irreversible factors such as age and comorbidity.

- We have demonstrated in a single centre retrospective study of more than 1200 patients with ESRF that a short duration of specialist predialysis follow up is associated with an increased risk of death on dialysis, principally during the first few months of treatment.

- Delays in the referral to renal specialist services of patients with advanced chronic renal failure should be minimised.

referral to renal specialist services of patients with advanced chronic renal failure should be minimised. Regular biochemical screening of high risk populations is probably the most effective way of ensuring timely specialist referral, but the additional resources that this requires will have implications for future planning and funding of renal services. ${ }^{18}$

We are grateful to Dr E J Will and Professor A M Davison for access to data regarding patients under their care, and for helpful discussion and advice. JS is supported by the Yorkshire Kidney Research Fund.

1 US Renal Data System. USRDS 1995 annual data report. Bethesda, MD: National Institutes of Health, National
Institute of Diabetes and Digestive and Kidney Diseases, April 1995.

2 Wolfe RA, Ashby VB, Milford EL, et al. Comparison of Wolfe RA, Ashby VB, Milford EL, et al. Comparison of
mortality in all patients on dialysis, patients on dialysis mortality in all patients on dialysis, patients on dialysis awaiting transplantation, and recipients of a

3 UK Renal Registry. Report 2000. Bristol: UK Renal Registry, 2000.

4 US Renal Data System. USRDS 1997 Annual data report.Bethesda, MD: National Institutes of Health, National Institute of Diabetes and Digestive and Kidney Diseases, 1997.

5 Feldman HI, Kinosian M, Bilker WB, et al. Effect of dialyzer reuse on survival of patients treated with hemodialysis. ҰAMA 1996;276:620-5.

6 Held PJ, Port FK, Wolfe RA, et al. The dose of hemodialysis and patient mortality. Kidney Int 1996;50:550-6.

7 Keane WF, Collins AJ. Influence of co-morbidity on mortality and morbidity in patients treated with hemodialysis. Am f Kidney Dis 1994;24:1010-18.

8 Arora P, Obrador GT, Ruthazer R, et al. Prevalence, predictors and consequences of late nephrology referral at a tertitors and consequences of late nephrology referral at

9 Hakim RM, Lazarus JM. Initiation of dialysis. $\mathscr{f} \mathrm{Am}$ Soc Nephrol 1995;6:1319-28.

10 Mitch WE, Maroni BJ. Nutritional considerations and the indications for dialysis. Am $\mathcal{F}$ Kidney Dis 1998;31:185-9.

11 Jungers $P$, Zingraff J, Albouze G, et al. Late referral to maintenance dialysis: detrimental consequences. Nephrol Dial Transplant 1993;8:1089-93.

12 Eadington DW. Delayed referral for dialysis. Nephrol Dial Transplant 1996;11:2124-6.

13 Eadington DW, Craig KJ, Winney RJ. Comorbidity and biochemical indices modulate the impact of late referral on survival on RRT. Nephrol Dial Transplant 1994;9:960-3.

14 Sesso R, Belasco AG. Late diagnosis of chronic renal failure and mortality on maintenance dialysis. Nephrol Dial Transplant 1996;11:2417-20.

15 Innes A, Rowe PA, Burden RP, et al. Early deaths on renal replacement therapy: the need for early nephrological referreplacement therapy: the need for early nep
ral. Nephrol Dial Transplant 1992;7:467-71.

16 Campbell JD, Ewigman B, Hosokawa M, et al. The timing of referral of patients with end-stage renal disease. Dial Transreferral of patients with

17 Ifudu O, Dawood M, Homel P, et al. Excess morbidity in patients starting uremia therapy without prior care by a nephrologist. Am $\mathcal{F}$ Kidney Dis 1996;28:841-5.

18 Khan IH, Catto GRD, Edward N, et al. Chronic renal failure: factors influencing nephrology referral. $Q f \mathrm{Med}$ 1994;87:559-64. 